\title{
Optimal Control of Quantum Rings by Terahertz Laser Pulses
}

\author{
E. Räsänen, ${ }^{1,3, *}$ A. Castro, ${ }^{1,3}$ J. Werschnik, ${ }^{1,3}$ A. Rubio, ${ }^{2,1,3}$ and E. K. U. Gross ${ }^{1,3}$ \\ ${ }^{1}$ Institut für Theoretische Physik, Freie Universität Berlin, Arnimallee 14, D-14195 Berlin, Germany \\ ${ }^{2}$ Departamento de Física de Materiales, Facultad de Químicas Universidad del País Vasco, Centro Mixto CSIC-UPV, \\ Donostia International Physics Center (DIPC), E-20018 Donostia-San Sebastián, Spain \\ ${ }^{3}$ European Theoretical Spectroscopy Facility (ETSF) \\ (Received 21 November 2006; published 13 April 2007)
}

\begin{abstract}
Complete control of single-electron states in a two-dimensional semiconductor quantum-ring model is established, opening a path into coherent laser-driven single-gate qubits. The control scheme is developed in the framework of optimal-control theory for laser pulses of two-component polarization. In terms of pulse lengths and target-state occupations, the scheme is shown to be superior to conventional control methods that exploit Rabi oscillations generated by uniform circularly polarized pulses. Current-carrying states in a quantum ring can be used to manipulate a two-level subsystem at the ring center. Combining our results, we propose a realistic approach to construct a laser-driven single-gate qubit that has switching times in the terahertz regime.
\end{abstract}

In recent years there has been wide interest in quantum control of nanoscale systems. One of the main motivations behind these studies arises from the possibilities of using tailored laser-pulse sequences for logic operations [1]. Semiconductor quantum dots and quantum rings (QRs) [2] are likely to play an important role in these far-reaching developments. Their atomlike properties together with a high flexibility in size and shape construct an ideal playground for quantum control.

A fundamental question in laser control of a general $N$-level quantum system is controllability, i.e., if the control target such as a certain eigenstate can be reached even in principle. The first steps toward laser control of QRs were recently taken by analyzing the current generation in mesoscopic rings by time-delayed linear pulses [3], and in narrow QRs subjected to circularly polarized laser pulses [4]. A similar approach has been used also to generate ring currents in circular biomolecules such as $\mathrm{Mg}$-porphyrin [5]. Quantum optimal-control theory (OCT) [6] is a powerful tool to find optimal laser pulses for controlling a quantum system. The iterative scheme developed within OCT [7] converges monotonically to an optimal laser pulse for reaching the prescribed target, such as a desired final quantum state, at the end of the pulse.

In this Letter we apply OCT to semiconductor QRs of finite ring width. We construct optimal two-component laser pulses that drive the $\mathrm{QR}$ from a given initial state to any predefined target state. These terahertz pulses generate the desired transitions in significantly shorter times and higher accuracies than previously used circularly polarized continuous waves (cw) of finite lengths. Finally, we sketch how the full control of current-carrying states in QRs enables the construction of a coherent laser-driven singlegate qubit.

The time evolution of our system is described by the time-dependent Schrödinger equation

$$
i \hbar \frac{\partial}{\partial t} \Psi(\mathbf{r}, t)=\left[\hat{H}_{0}-\hat{\mu} \boldsymbol{\epsilon}(t)\right] \Psi(\mathbf{r}, t),
$$

where $\boldsymbol{\epsilon}(t)=\left(\epsilon_{x}(t), \boldsymbol{\epsilon}_{y}(t)\right)$ is the two-component laser field propagating in the $z$ direction. The interaction between the field and the electron is modeled in the dipole approximation (length gauge) with the dipole operator $\hat{\mu}=-e \mathbf{r}$. The static effective Hamiltonian for the semiconductor QR located on the $x y$ plane is written as

$$
\hat{H}_{0}=-\frac{\hbar^{2}}{2 m^{*}} \nabla^{2}+\frac{1}{2} m^{*} \omega_{0}^{2} r^{2}+V_{0} e^{-r^{2} / d^{2}},
$$

where the potential part with $r^{2}=x^{2}+y^{2}$ consists of a parabolic confinement and a Gaussian peak located at the center. Together they determine the shape of a twodimensional QR which is visualized in Fig. 1. We choose the parameter values $\hbar \omega_{0}=10 \mathrm{meV}, V_{0}=200 \mathrm{meV}$, and $d=10 \mathrm{~nm}$ in order to follow the energy and length scales of $\mathrm{QR}$ experiments [2]. The parameters yield a ring radius of $r_{0} \approx 22 \mathrm{~nm}$. The effective mass of the electron in GaAs semiconductor medium is $m^{*}=0.067 m_{e}$. Using the dielectric constant $\kappa=12.7 \epsilon_{0}$ for GaAs, the effective atomic units [marked as (a.u.) in the results below] scale as $\mathrm{Ha}^{*}=\left(m^{*} / \kappa^{2}\right) \mathrm{Ha} \approx 11.30 \mathrm{meV}, a_{B}^{*}=\left(m^{*} / \kappa\right) a_{0} \approx$ $10.03 \mathrm{~nm}$, and $u_{t}^{*}=\hbar / \mathrm{Ha}^{*} \approx 58.23 \mathrm{fs}$.

Figure 1(b) shows the energy-level spectrum for nine lowest states $\psi_{j}^{l}$, where $l$ is the angular momentum and index $j=|l|+1$ enumerates the levels in energy. Because of the finite width of the ring, there are other radial bands which, however, appear at higher energies; e.g., the second band appears at $E=5.03$. The selection rules allow transitions only between states with consecutive angular momenta $l=\ldots,-2,-1,0,1,2, \ldots$, i.e., along the dashed line marked in the figure. The transition probabilities between the levels can be calculated as $P_{j k}=\left|\left\langle\psi_{k}|\hat{\mu}| \psi_{j}\right\rangle\right|^{2}$. For the first levels shown in Fig. 1(b) we get relative 
(a)

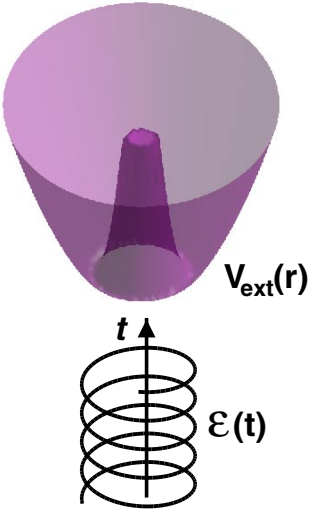

(b)

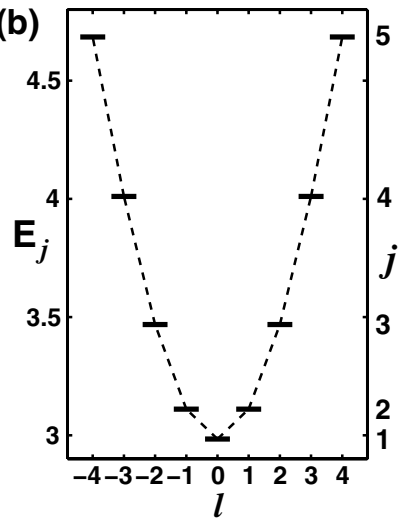

FIG. 1 (color online). (a) Shape of the external confining potential for a quantum ring and an example of a circularly polarized laser field. (b) Energy-level spectrum of a quantum ring. The transitions are allowed along the dashed line so that $\Delta l= \pm 1$.

transition probabilities $P_{12}=1, P_{23}=1.04, P_{34}=1.12$, and $P_{45}=1.24$. Hence, the two-dimensional nature of the ring is reflected also in the varying $P_{j k}$ : in one-dimensional rings, the dipole matrix elements are constant along the transition lines [4].

We apply OCT to find optimal laser pulses for population transfer from the initial state $\Phi_{i}=\Psi(t=0)$ to the target state $\Phi_{f}$, so that both of these states are preselected from the QR eigenstates $\psi_{j}^{l}$. In OCT formalism we thus use the projection operator $\left|\Phi_{f}\right\rangle\left\langle\Phi_{f}\right|$ as the target operator, whose expectation value is maximized at the end of the pulse $(t=T)$. This corresponds to maximizing the overlap

$$
J_{1}[\Psi]=\left\langle\Psi(T) \mid \Phi_{f}\right\rangle\left\langle\Phi_{f} \mid \Psi(T)\right\rangle=\left|\left\langle\Psi(T) \mid \Phi_{f}\right\rangle\right|^{2} .
$$

We also require that the fluence (time-integrated intensity) of the laser pulse is as small as possible by minimizing the functional

$$
J_{2}[\epsilon]=-\int_{0}^{T} d t \alpha(t)\left[\epsilon_{x}^{2}(t)+\epsilon_{y}^{2}(t)\right],
$$

where the predefined function $\alpha(t)$ acts as a penalty factor, which allows us to impose constraints on the envelope function of the laser pulse [8]. We can apply, e.g., sinusoidal envelope functions as demonstrated in Ref. [9]. However, in the examples below we have set $\alpha=1$ (rectangular pulses) in order to simplify the comparison between OCT and cw approaches. As the second constraint, the wave function and its complex conjugate need to satisfy the time-dependent Schrödinger equation. This condition is expressed in a functional form (in a.u.) as

$$
J_{3}[\epsilon, \Psi, \chi]=-2 \operatorname{Im} \int_{0}^{T} d t\left\langle\chi(t)\left|\left(i \partial_{t}-\hat{H}(t)\right)\right| \Psi(t)\right\rangle,
$$

where $\chi(t)$ is the Lagrange multiplier. Now, the minimum of the total Lagrange functional $J=J_{1}+J_{2}+J_{3}$ can be determined by setting the variations with respect to $\Psi, \epsilon$,

and $\chi$ independently to zero. This yields a set of control equations

$$
\begin{gathered}
i \partial_{t} \Psi(t)=\hat{H} \Psi(t), \quad \Psi(0)=\Phi_{i}, \\
i \partial_{t} \chi(t)=\hat{H} \chi(t), \quad \chi(T)=\Phi_{f}\left\langle\Phi_{f} \mid \Psi(T)\right\rangle, \\
\epsilon(t)=-\frac{1}{\alpha} \operatorname{Im}\langle\chi(t)|\hat{\mu}| \Psi(t)\rangle,
\end{gathered}
$$

which can be solved iteratively in order to find the optimized field $\epsilon(t)$ at the end of the procedure [7]. For the forward and backward propagation of $\Psi(t)$ and $\chi(t)$, we apply a rapidly converging scheme discussed in Refs. [7,9]. This computational scheme has been recently included in the OCTOPUS code [10].

The advantage of OCT becomes obvious already in the simplest transition from $\psi_{1}^{0}$ to $\psi_{2}^{1}$ (denoted below as $|1\rangle \rightarrow$ $|2\rangle)$. Figure 2(a) shows the $x$ and $y$ components of the optimized pulse when the pulse length is fixed to $T=$ 100 ( $\sim 5.8 \mathrm{ps}$ ) and the penalty factor is $\alpha=1$. In comparison, the dashed lines show the cw: $\epsilon_{\mathrm{cw}}(t)=$ $A[\cos (\omega t) \hat{x} \pm \sin (\omega t) \hat{y}]$, where \pm denotes $\sigma_{ \pm}$polarization, and $A$ and $\omega$ are the amplitude and frequency of the field, respectively. Here we choose $\omega=\omega_{12}$ and $A=$ $\Omega_{R} / \mu_{12}=\pi / \mu_{12} T$, where $\Omega_{R}$ is the Rabi frequency. These parameters correspond to the $\pi$-pulse condition. The cw leads to an occupation of 0.99 in the second state which, as demonstrated below, is insufficient in terms of the controllability of the QR. In contrast, the optimized pulse produces a value 0.9998 for the same pulse length. The underlying reason for the difference becomes evident from the spectrum of the optimized pulse shown in Fig. 2(b). Namely, the pulse contains a considerable fraction of the next eigenfrequency $\omega_{23}$, preventing the population flow into the third state. If the system is subjected to a cw instead, the occupation loss is inevitable, and becomes pronounced at shorter pulse lengths when the amplitudes are correspondingly higher.
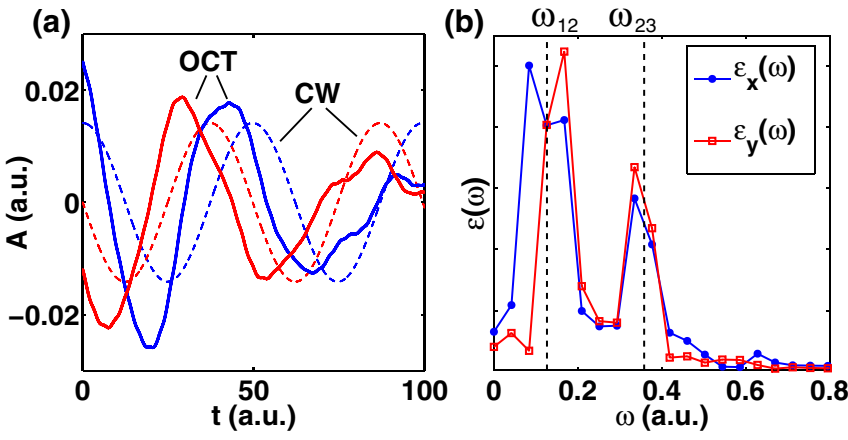

FIG. 2 (color online). (a) Components $\epsilon_{x}(t)$ [blue (dark gray)] and $\epsilon_{y}(t)$ [red (light gray)] of the optimized (solid lines) and uniform laser pulses (dashed lines) for $|1\rangle \rightarrow|2\rangle$ transition. (b) Spectrum of the optimized field. The dashed lines correspond to eigenfrequencies $\omega_{12}$ and $\omega_{23}$. 
In Fig. 3 the yields of OCT and cw approaches for the $|1\rangle \rightarrow|2\rangle$ transition are compared for different pulse lengths in a logarithmic scale. Considering the required pulse lengths for desired occupation accuracies, OCT is clearly superior to the $\mathrm{cw}$ approach. The relation between the pulse lengths of these methods $T_{\mathrm{cw}} / T_{\mathrm{OCT}}$ for a certain occupation always doubles when the accuracy is increased by an order of magnitude. For example, the occupation of 0.99 requires $T_{\mathrm{cw}} \sim 100$ or $T_{\mathrm{OCT}} \sim 25$, whereas for an accuracy of 0.999 the $\mathrm{cw}$ needs to be increased up to $T_{\mathrm{cw}} \sim$ 300 and the optimized pulse only to $T_{\mathrm{OCT}} \sim 35$. The main benefit in very high occupations achieved by short optimized pulses is the ability to perform a large number of successive operations (see below). In single transitions we can qualitatively assess the required occupation accuracies by checking the final densities of the propagated wave function. The insets of Fig. 3 reveal that occupations close to 0.999 are required for the target states in order to preserve a reasonable circular symmetry of the wave function.

The benefits of OCT become even more pronounced in multilevel transitions. As shown above, there is only a single excitation path in the lowest radial band of our QR. The path is subjected to strict selection rules that allow transitions only between consecutive levels with $\Delta l= \pm 1$. Hence, in the cw scheme the intermediate states require full population before occupation of the final state. Eventually this leads to very slow transition processes sensitive to external perturbations of the real device. In OCT approach instead, full population of the intermediate states is not needed, since the components in the optimized pulse drive the occupation continuously toward the target state, still obeying the selection rules. For example, in

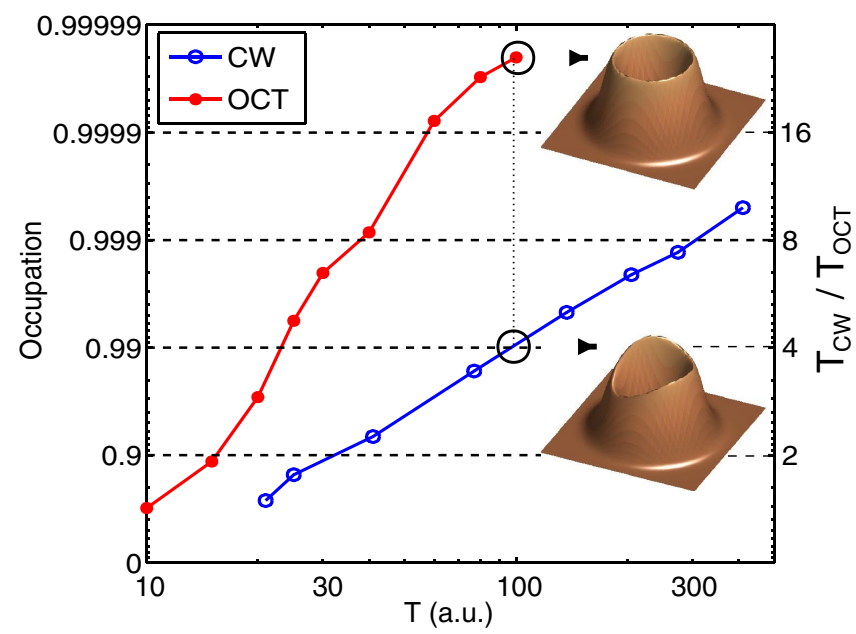

FIG. 3 (color online). Maximum occupation of the target state in transition $|1\rangle \rightarrow|2\rangle$ as a function of the pulse length. The open (blue) circles correspond to continuous waves and the filled (red) circles to the optimal-control result. The insets show the densities $|\Psi(T=100)|^{2}$ when the corresponding achieved occupations are 0.99 and 0.9998 for these pulse types, respectively. $|1\rangle \rightarrow|4\rangle$ transition the maximum occupation required for the intermediate states $|2\rangle$ and $|3\rangle$ is $\sim 60 \%, \ldots, 80 \%$, depending on the pulse length (here $T=50, \ldots, 200$ ).

The chirality of a QR state can be changed in multilevel transitions from $l$ to $-l$ (or vice versa). Figure 4 shows schematic pictures (upper panel) of transitions from $\psi_{2}^{-1}$ to $\psi_{2}^{1}$ (a) and from $\psi_{3}^{-2}$ to $\psi_{3}^{2}$ (b). The optimized pulses given in the middle panel of Fig. 4 are approximately symmetric around the midpoints at $t=T / 2$, and at this point the polarization direction of the field changes from $\sigma_{+}$to $\sigma_{-}$. Hence, a backward process can be obtained by applying a pulse where the $x$ and $y$ components are exchanged.

The intermediate states, which in this case contain the ground state with $l=0$, require only partial occupation in the transition process. The intermediate occupations shown in the lower panel of Fig. 4 reach a maximum of $\sim 60 \%$ in both $l=-1 \rightarrow 1$ and $l=-2 \rightarrow 2$ transitions. Similar to the pulse shapes, the occupation curves are approximately symmetric around $t=T / 2$. The final occupations of the target states are 0.998 and 0.996 , respectively.

In Fig. 5 we plot the time-dependent electron densities $|\Psi(\mathbf{r}, t)|^{2}$ (color scale) and the current densities $\mathbf{j}(\mathbf{r}, t)=$ $i \hbar\left(\Psi \nabla \Psi^{*}-\Psi^{*} \nabla \Psi\right) / 2 m^{*}$ (arrows) at different times in the transition from $l=-1$ to $l=1$, corresponding to Fig. 4(a). The circularly symmetric initial state in 5(a) carries a counterclockwise current of $I \approx 0.11 \mu \mathrm{A}$. During the propagation, the density and current localize first to a single maximum [Fig. 5(b)]. At the midpoint [Fig. 5(c)] there are two density maxima along the ring and opposite currents between them along the two paths, arising from the partial occupations of $l= \pm 1$. The net current is zero at this point. As expected, the final state
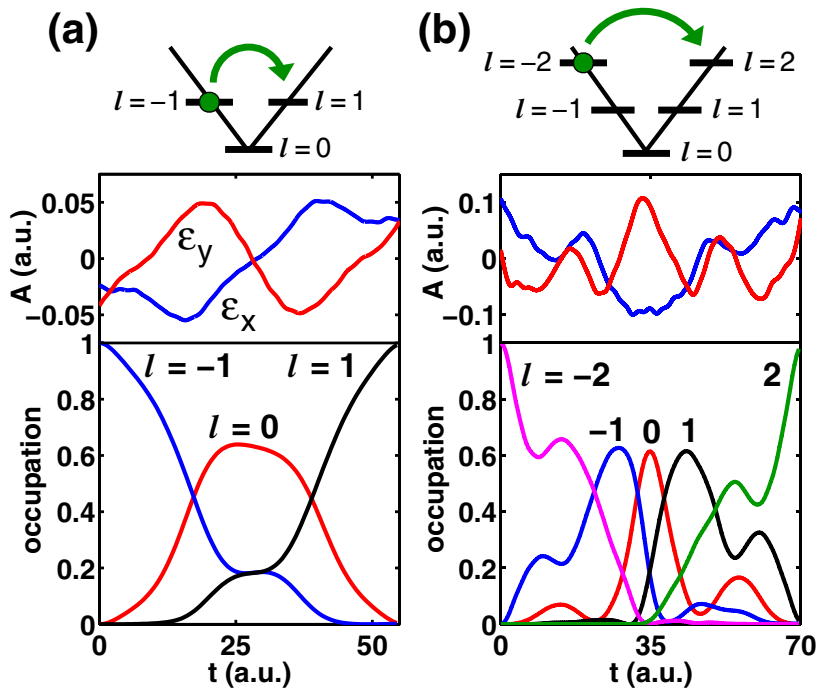

FIG. 4 (color online). Schematic picture of transitions from $l=-1$ to $l=1$ (a) and from $l=-2$ to $l=2$ (b) (upper panel), optimized fields for these transitions (middle panel), and the occupations of the states (lower panel). 

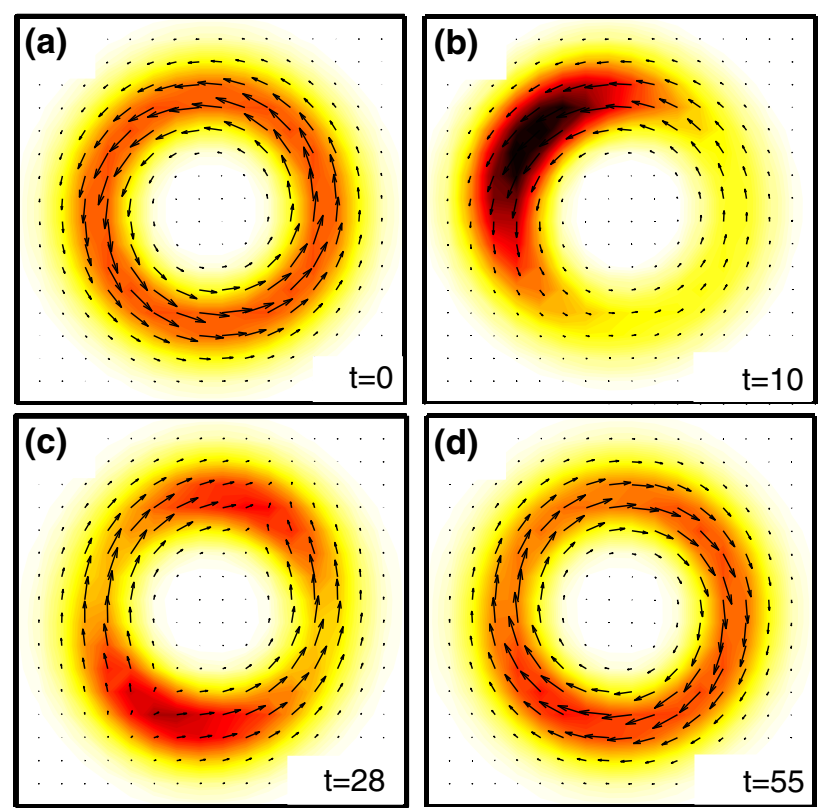

FIG. 5 (color online). Time-dependent electron density $|\Psi(\mathbf{r}, t)|^{2}$ [light shade (yellow), low; black, high] and the current $\mathbf{j}(\mathbf{r}, t)$ (arrows) at different times in the transition from $l=-1$ to $l=1$ driven by the optimized pulse [see Fig. 4(a)].

reached at $T=55$ [Fig. 5(d)] has a symmetric clockwise current.

The circular currents in QRs induce magnetic fields through the ring in accordance with the Biot-Savart law. The current-flipping processes correspond to changes in magnetic fields as $B \hat{z} \leftrightharpoons-B \hat{z}$ at the center of the ring, where $B=\mu_{0} I / 2 r_{0} \approx 3 \mu \mathrm{T}$ for a single electron occupying the state $l= \pm 1$. If needed, higher fields can be obtained by occupying higher states, by decreasing the ring size, or by changing the semiconductor host material.

Combining the results presented above, we suggest the following single-qubit gate construction. First, the accurate experimental shape of the QR and thus the static Hamiltonian (2) have to be determined in order to allow for a full control. This can be done, e.g., by tuning the potential parameters to reproduce the single-electron spectrum obtained in a transport measurement [11]. Second, the $\mathrm{QR}$ is controlled by a sequential master pulse achieving first the initial excitation from $l=0$ to $l=l_{m}$ (qubit initialization) and then performing $l_{m} \leftrightharpoons-l_{m}$ operations at desired times so that the opposite operation is obtained by exchanging $\epsilon_{x}$ and $\epsilon_{y}$ components of the currentflipping pulse. The magnetic field induced at the ring center changes the spin state (up or down) of an applicable subsystem, e.g., a magnetic particle [4] or an attached quantum dot. This is feasible within our picosecond-scale gate operation times, since the characteristic relaxation times are of the order of nanoseconds [4], and the decoherence times in quantum dots (of similar physical parameters) have been measured to be on the millisecond scale
[12]. Actual quantum computation would still require a two-qubit gate and a stable readout scheme, but these developments, as well as detailed experimental considerations, are beyond the scope of this study. However, we point out that terahertz frequency regime is routinely reached by, e.g., quantum cascade lasers, and the technology of pulse refinement is under rapid development [13].

In conclusion, we have shown that a complete control of realistic single-electron quantum rings can be obtained using (quantum) optimal-control theory. The optimized pulses perform the desired operations at significantly shorter times and better accuracies than the continuous waves, and in multilevel transitions they are not restricted by the need to fully populate the intermediate states. This enables a simple construction of a pulse that coherently flips the spin of a subsystem at the center of the ring at preselected times.

This work was supported by the EU's Sixth Framework Programme through the Nanoquanta Network of Excellence (No. NMP4-CT-2004-500198), SANES project (No. NMP4-CT-2006-017310), DNA-NANODEVICES (No. IST-2006-029192), BSC (Barcelona Mare Nostrum Center), the Humboldt Foundation, the Academy of Finland, the Finnish Academy of Science and Letters through the Viljo, Yrjö, and Kalle Väisälä Foundation, and the Deutsche Forschungsgemeinschaft through SFB 658.

*Electronic address: esa@physik.fu-berlin.de

[1] See, e.g., D.P. Di Vincenzo and C.H. Bennett, Nature (London) 404, 247 (2000).

[2] For a review on quantum rings, see, e.g., T. Ihn et al., Europhysics News 36, 78 (2005); S. Viefers et al., Physica (Amsterdam) 21E, 1 (2004).

[3] A. Matos-Abiague and J. Berakdar, Phys. Rev. Lett. 94, 166801 (2005).

[4] Y. V. Pershin and C. Piermarocchi, Phys. Rev. B 72, 245331 (2005).

[5] I. Barth et al., J. Am. Chem. Soc. 128, 7043 (2006); I. Barth and J. Manz, Angew. Chem., Int. Ed. 45, 2962 (2006).

[6] A. P. Peirce, M. A. Dahleh, and H. Rabitz, Phys. Rev. A 37, 4950 (1988); R. Kosloff et al., Chem. Phys. 139, 201 (1989).

[7] W. Zhu and H. Rabitz, J. Chem. Phys. 109, 385 (1998).

[8] K. Sundermann and R. de Vivie-Riedle, J. Chem. Phys. 110, 1896 (1999).

[9] J. Werschnik, Quantum Optimal Control Theory: Filter Techniques, Time-Dependent Targets, and TimeDependent Density-Functional Theory (Cuvillier, Göttingen, 2006).

[10] A. Castro et al., Phys. Status Solidi B 243, 2465 (2006).

[11] E. Räsänen et al., Phys. Rev. B 70, 115308 (2004).

[12] H. A. Engel et al., Quant. Info. Proc. 3, 115 (2004).

[13] M. Tonouchi, Nature Photonics 1, 97 (2007). 